\title{
Time Slots Investment of a dead node in LEACH protocol on Wireless Sensor Network
}

\author{
Abedelhalim HNINI \\ LAVETE laboratory Mathematics and \\ Computer Science Dept Sciences and Tech Fac \\ Settat, 26000, Morocco \\ FIHRI Mohammed \\ LAVETE laboratory Mathematics and \\ Computer Science Dept Sciences and Tech Fac \\ Settat, 26000, Morocco
}

\author{
Abdellah EZZATI \\ LAVETE laboratory Mathematics and \\ Computer Science Dept Sciences and Tech Fac \\ Settat, 26000, Morocco \\ Abdelmajid HAJAMI \\ LAVETE laboratory Mathematics and \\ Computer Science Dept Sciences and Tech Fac \\ Settat, 26000, Morocco
}

\begin{abstract}
Wireless sensor network (MSN) is a wirelessly interconnected network. WSN promises a wide range of potential such as surveillance, military and civilian, to name just a few, applications. A sensor node senses the environment and delivers data to the sink. Energy saving is one of the keys to challenge network life time. LEACH protocol has been incorporated to extend network life time. This protocol forms clusters of the sensor nodes and elect one of them to become a Cluster Head (CH) to route data cluster to sink. In cluster, communication uses TDMA technique. This latter organizes transmission time (Time Slot) which corresponds to each node member of cluster. When a node dies, time slot corresponding to this node will be free. In this paper, we will present LEACH comportments after a node dies, then we will propose some ideas to invest its time slots by alive node members to maximize data reception time parameter so throughput end-to-end. This parameter is very important for real-time data. Finally, we will simulate this idea with Network Simulator (NS2) to argue for our propositions.
\end{abstract}

Keywords-Network Clustering; routing protocol; Ad hoc Network; LEACH; WSN; Node dies

\section{INTRODUCTION}

Wireless Sensor Networks (WSNs) have gained booming interest in recent years. They are used in various fields: military, agriculture, meteorology and medicine... Wireless Sensor Network consists of a huge number of nodes called sensor nodes. They are deployed in a spacious area. A typical sensor node is made of 4 building blocks: power unit, communication unit, processing unit and sensing unit. Limited network life is one of the most critical inconvenience and limitations of WSNs. It's arduous to recharge or to change batteries in the battery-powered sensor nodes. That is the reason why many researchers have been incorporated to increase the network life time.

The clustered WSN compromises three types of entities: the base station, cluster head sensor node and non-cluster head sensors. Non-cluster head sensor nodes take the information and send it to the respective cluster head. This latter sends the data to the base station directly in order to resolve the life time problem, which's due to the fact that when a battery node dies, it becomes useless. Several protocols have been used for a long time. Hierarchical routing protocols are the best used protocols to maximize the network life time. LEACH protocol is one of the fundamental protocols that can be used to decrease the energy which is consumed to aggregate and send the data. Each node has a time slot of data transmission, even the dead nodes or the nodes outside the area. In our contribution, we seek to invest the free slots of the dead nodes.

Our work is organized in the following way: section 1 introduces the WSNs. Section 2 presents related works on the hierarchical routing protocol and the existing LEACH descendent. Section 3 includes the presentation of our contribution. Section 4 shows the simulation steps and demonstrates the simulation results. The last section conclues our work.

\section{RELATED WORKS}

LEACH protocol is a basic hierarchical protocol for the WSN. It's made up of nodes cluster. Each cluster has a special node as cluster head. This latter collects data from nodes that belong to the respective cluster and transmits it to the base station [1][2].

LEACH protocol has two phases, the set up phase and the steady state phase. In set up phase, the clusters are formed and the cluster heads are chosen. Each node decides if it will become a cluster head. The decision is made by the node selecting a stochastic number between 0 and 1 . If the number is less than a threshold $\mathrm{T}(\mathrm{n})$, the node becomes a cluster head for the current round [1].

The threshold is set as:

$$
\mathrm{T}(\mathrm{n})=\left\{\begin{array}{c}
\frac{\mathrm{p}}{1-\mathrm{p} *\left[\mathrm{r} \bmod \left(\frac{1}{\mathrm{p}}\right)\right]}, \mathrm{n} \in \mathrm{G} \\
0, \quad \text { otherwise }
\end{array}\right.
$$

Where:

- $\mathrm{p}$ is the probability of needed being selected as a cluster head node; 
- $\mathrm{r}$ is the number of rounds passed;

- $\mathrm{G}$ is the collection of ordinary nodes mod denotes modulo operator.

In the steady state phase the cluster head is maintained and the data is transmitted between nodes. The cluster head sends all the data to the base station after receiving and aggregating it. Each cluster communicates using the TDMA technique, which divides time of communication by slots, each time slot corresponds to a node of the cluster that can deliver data in their time slot.

A node is free only when a node is out of area or dead.

A modified version of the LEACH protocol is known as the LEACH-C [2], we call it also LEACH Centralized. This protocol uses the same steady-state phase as the LEACH. However, in set-up phase each node omits information concerning its current position, which is determined using position finding system, and residual energy level to the base station.

Another extension of LEACH is the TEEN (thresholdsensitive Energy Efficient sensor Network), in which two thresholds are added for the sensed attribute: the hard threshold and the soft one. The hard threshold is the minimum value of the sensed attribute required to oblige a sensor node to activate its transmitter and transmit to the cluster head. The soft threshold emitted by the cluster head to its nodes member and the cluster configuration. All this is done to increase the life span of network [3].

Most studies have shares on LEACH protocol and its descendants interesting by life time protocol but most of these works are not interested in the freedom of time slots which corresponds to the dead node.

LEACH-V [4] is introduced to overcome the problem when a $\mathrm{CH}$ dies or does not have sufficient energy to transmit the received data arriving from cluster members to the base station. In this case, another member node will become a $\mathrm{CH}$ of the cluster. This node is called vice cluster head. This one is interested in the death of $\mathrm{CH}$ but it doesn't also use the free time slots corresponding to the dead node member.

The exception studies are interested in a phenomenon which is close to free time slots is presented in [5][6][7][8], all these studies meet the influence mobility of nodes in term of packet loss. But there added a lot of flow to control packet loss. Therefore they consume a lot of energy. . In addition, they took the packet loss even if a node that does not have any data to transmit, it changed the cluster or its battery is sold out.

All this protocol, when a member node (not $\mathrm{CH}$ ) dies, the time slot reserved of this node is free or it is using with more energy . That's what we did to develop this free time to invest it and use it by other node, in a manner minimizing power consumption.

\section{CONTRIBUTION}

LEACH protocol divides sensor network to form clusters and randomly selects a $\mathrm{CH}$ (Cluster Head) in each cluster. In a cluster member node, which is not a $\mathrm{CH}$, senses data and transmits it to the $\mathrm{CH}$. This latter aggregates the received data and forwards these data to base station. As presented in schema.

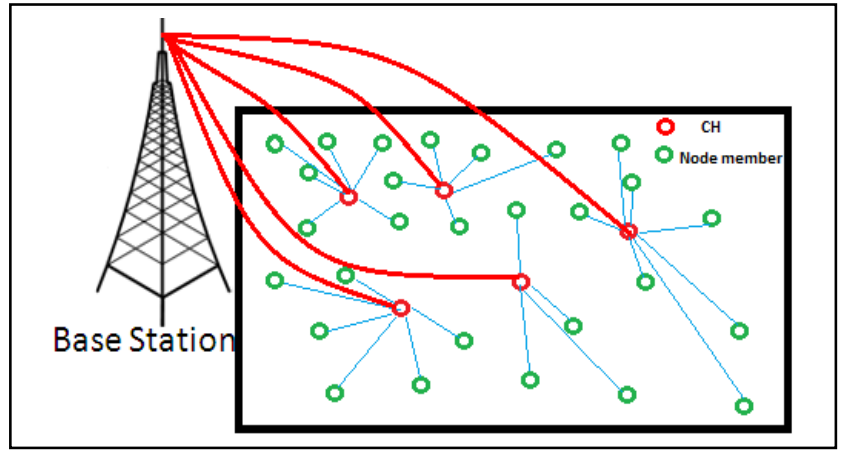

Fig. 1. Clustering in LEACH

In cluster, communication between $\mathrm{CH}$ and its member nodes use TDMA technique to synchronize data transmission time. TDMA divides time of communication to form frames. This latter is subdivided to form Time Slot: data transmission time corresponds to one member node. Every node has one time slot as presented in figure (figure 1).

\section{A. The energy dissipation of the steady data transmission phase}

A sensor uses its energy to realize three main activities: the acquisition, communication and data processing.

Acquisition: the energy consumed to complete the acquisition is not very important. However it depends on the phenomenon and the type of monitoring performance.

Communication: The communications consumes a lot of energy than other spots. They cover communication transmission and reception.

Treatments: the energy consumed in calculations is much

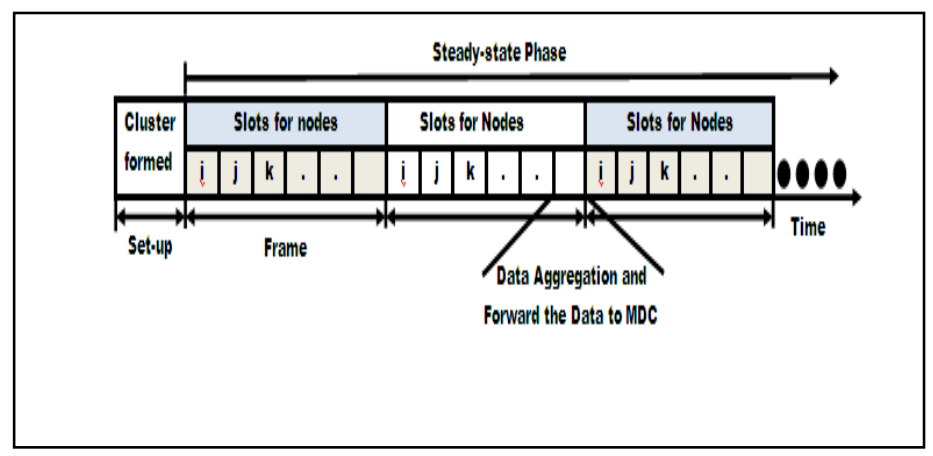

Fig. 2. chronologique communication in LEACH

lower than the energy of communications.

In $\mathrm{LEACH}$ [2], to transmit a K-bit message for a distance d. the transmission consumes energy as showed below:

$\operatorname{ETx}(\mathrm{K}, \mathrm{d})=$ Eelec $\times \mathrm{k}+$ eamp $\times \mathrm{k} \times \mathrm{d} 2$

To receive a K-bit message the reception consumes:

$\operatorname{ERx}(\mathrm{K})=\operatorname{ERx}-$ elec $(\mathrm{k})=$ Eelec $\times \mathrm{k}$ 
Where Eelec: energy transmission/ reception power.

$\mathrm{K}$ : message size

$\mathrm{d}$ : the distance between transmitter and receiver.

eamp: amplification factor.

Each cluster head dissipates energy among the signals received from the nodes and transmits the aggregated signals to the BS since the BS is far from the nodes. Presumably, the energy dissipation follows (d4 power loss); therefore the energy dissipated in the cluster head node during a single frame is:

$$
\begin{aligned}
& \text { Eframe }=N / k *(\operatorname{ETx}(K, d)+\operatorname{ERx}(K))+\text { Ech } \\
& \text { Ech } \quad=N / k * \operatorname{ERx}(K)+N / k * \operatorname{ETx}(K, d 2)
\end{aligned}
$$

Where we assume that there are $\mathrm{N}$ nodes distributed uniformly in an $\mathrm{M}^{*} \mathrm{M}$ region and there are k clusters, there is an average of $\mathrm{N} / \mathrm{k}$ nodes per cluster.

Each non-cluster head node needs less energy to transmit its data to the $\mathrm{CH}$. Thus the energy used in each non cluster head node is:

$$
\operatorname{EnotCH}=\operatorname{ETx}(K, d)+\operatorname{ERx}(K)
$$

Where $\mathrm{d}$ is the distance from the node to $\mathrm{CH}$.

\section{B. The communication technique inside one cluster}

In this paper, we will do a restatement of slots. We distribute free time slot of the dead nodes to the other living nodes with regard to three different possible cases. In the first case, the free time slot will be given to the first node in the list. In the second, it will be given to the last node as $\mathrm{CH}$. In the last case, in every turn, we reserve it for a member node in the cluster.

In the first case, the free time slot is reserved to the first node in cluster as showed in figure 3 . We assume that node N3 is dead after Frame 1, so the organization of time slots will change according to the diagram in Figure 3. After the end of each frame $\mathrm{CH}$ informs the first node in cluster (N2) to lock this free time slot. Energy dissipation is added to the equation (3) as Esprending to inform node N1 so (4) revert equation (7)

$$
\text { Eframe }=\mathrm{N} / \mathrm{k} *(\operatorname{ETx}(\mathrm{K}, \mathrm{d})+\operatorname{ERx}(\mathrm{K}))+\mathrm{Ech}+
$$
Esprending ----- (7)

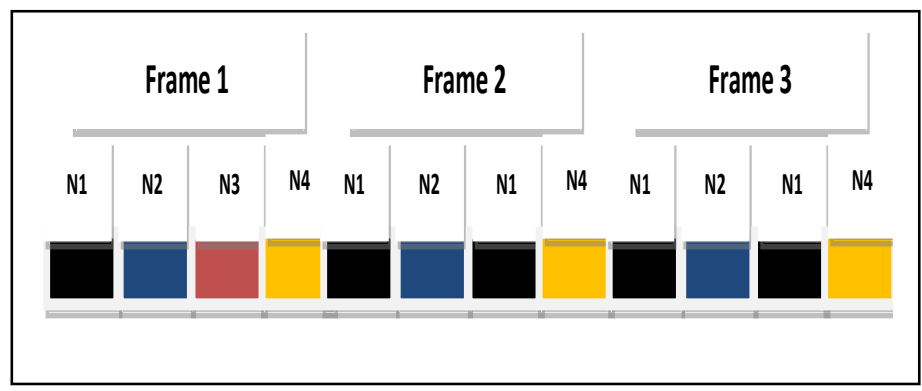

Fig. 3. The organization of the frame after the node N3 dies

In the second case, the free time slot is reserved to the last node in cluster that is the $\mathrm{CH}$. So after node N3 dies, we obtain the following organization (fig 4). We get the energy Esprending dissipated in (7) but $\mathrm{CH}$ always remains active to receive the data sent by their member nodes, so the $\mathrm{CH}$ dies earlier than the other nodes in the cluster.

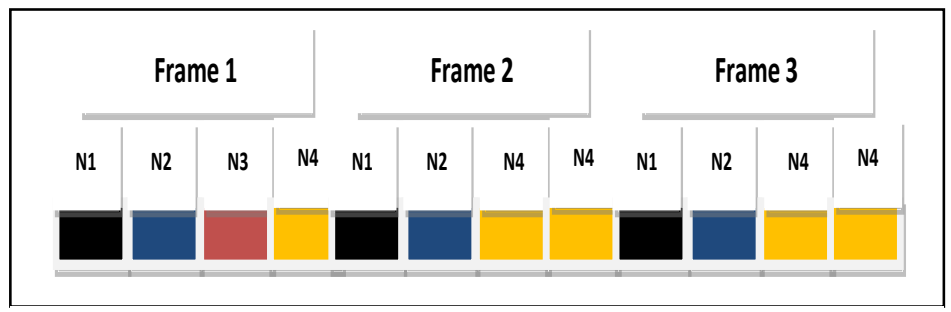

Fig. 4. The organization of free time slot to $\mathrm{CH}$

In the last case, in each frame we reserve the free time slot to one node in the cluster. As presented in fig 5. In this case, all the cluster nodes will be compelled, at the end of each frame, to be active to receive the broadcast message by the $\mathrm{CH}$ which reorganizes the time slots again. Energy dissipation is added to the equation (3) as Esprending $+\mathrm{k} / \mathrm{N}^{*}$ Eactive. So (4) change to (8)

Eframe $=\mathrm{N} / \mathrm{k} *(\operatorname{ETx}(\mathrm{K}, \mathrm{d})+\operatorname{ERx}(\mathrm{K}))+\mathrm{Ech}+$ Esprending $+\mathrm{k} / \mathrm{N} *$ Eactive ----- $(8)$.

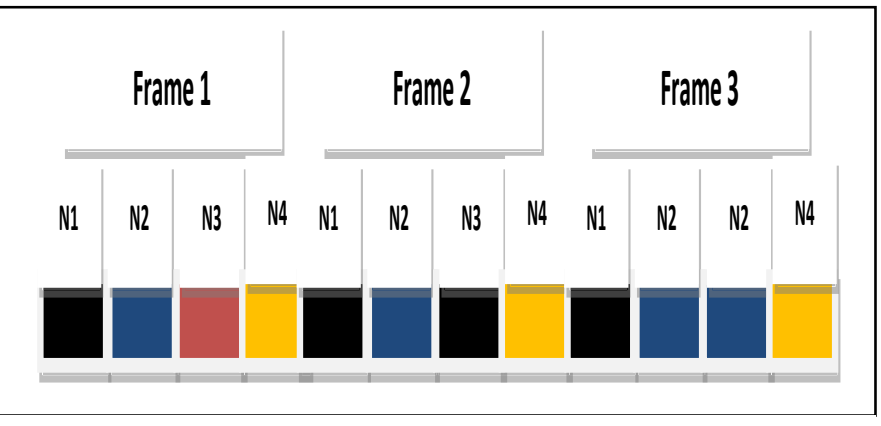

Fig. 5. In each frame free time slot reserved to one node in cluster

In all cases, we spend slightly more energy but we minimize the data time arrival, we maximize data aggregation and throughput. As a result, we maximize QoS parameter. These parameters will be presented clearly in the simulation result. WSN generates different traffic. This requires the classification of different type of services. In addition, real-time data normally demands larger bandwidth and entails higher network throughput to transport large volume of data to remote data sink rapidly and reliably. However, in this simulation we will focus on data reception rates, jitter and end-to-end reception. These parameters have a lot of benefits despite consuming a little bit more energy.

\section{Simulation Results}

We run our technique using NS-2 simulator (version 2.34) [13] with LEACH protocol [14] to determine its benefits. Then we will compare it to LEACH in terms of Throughput relative to the number of the alive nodes (data received to the BS in terms of number of the alive nodes per time), energy dissipation per time. For the experiment, we choose the following parameters: a network of 100 homogeneous nodes randomly deployed in a zone with dimensions of $1000 * 1000$ and a base station which is located at $(X=70, Y=200)$. For our evaluations, we use the average of 10 runs for each set of tested 
parameters and we neglect the case of the rotation of free time slots by living cluster nodes.

- Energy dissipation.

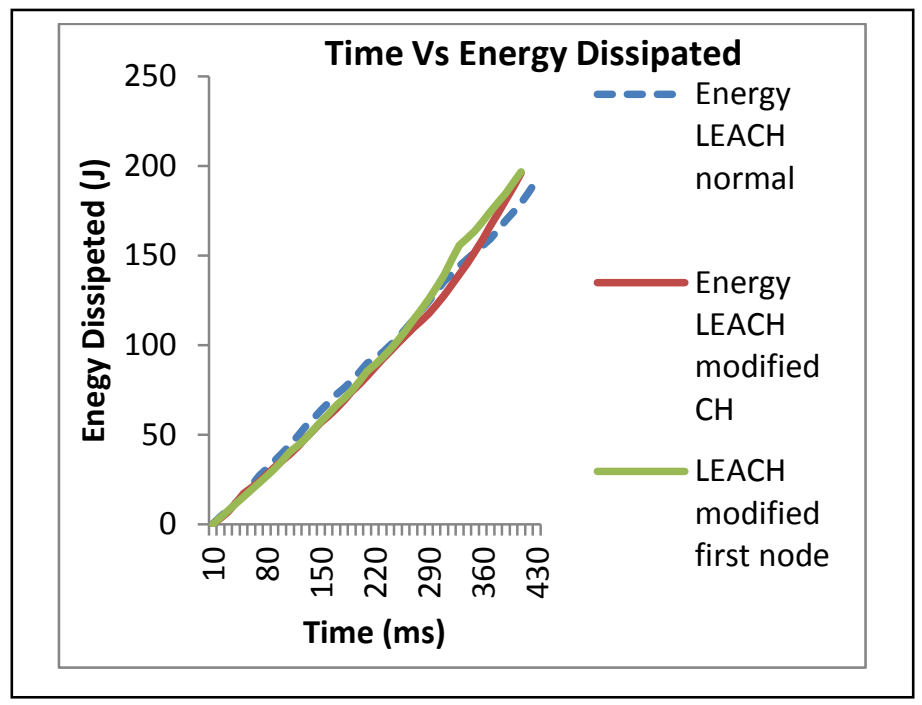

Fig. 6. Comparison of Energy Dissipated with Time

The first graph (Fig 6) in this figure depicts the energy dissipation of normal LEACH protocol and LEACH protocol with reformation of time slot. We see that after the time exceeds almost 200, the two lines \{LEACH is modified with free time slots reserved to $\mathrm{CH}$ (LEACH modified $\mathrm{CH}$ ), LEACH is modified with free time slot reserved to the first node in the cluster (LEACH modified first node) $\}$ are divergent of LEACH classic (LEACH normal) because it is time that the nodes begin to be exhausted which generates the free time slots that are used again later in LEACH modified by other nodes. That is why the lines of LEACH modified are superior to LEACH normal with reformation of time slots. But that is normal because we added a traffic signal by $\mathrm{CH}$ soon as the death of node for LEACH modified $\mathrm{CH}$ and the first node of threshold for LEACH modified first node. The performance is obvious at the end-to-end data aggregation depends on the alive member nodes and throughput of data reception.

- Average Throughput.

The second graph (Fig 7) shows the average throughput depending on time. In both cases, normal LEACH and LEACH with integration of our contribution, after the time exceeds almost 200, the two last lines have more throughput than the first line. This shows that the performance increases at the reception data then throughput. It is very clear in graph (Fig 8), when the nodes begin to die; the lines of LEACH modified are above the line of normal LEACH.

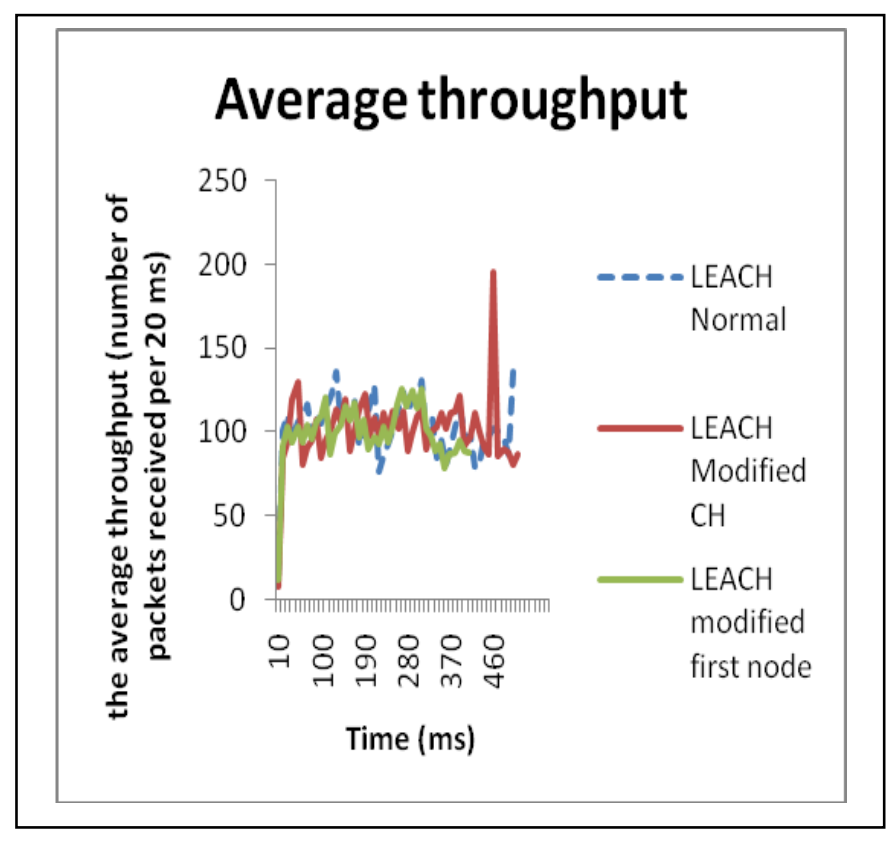

Fig. 7. The average throughput

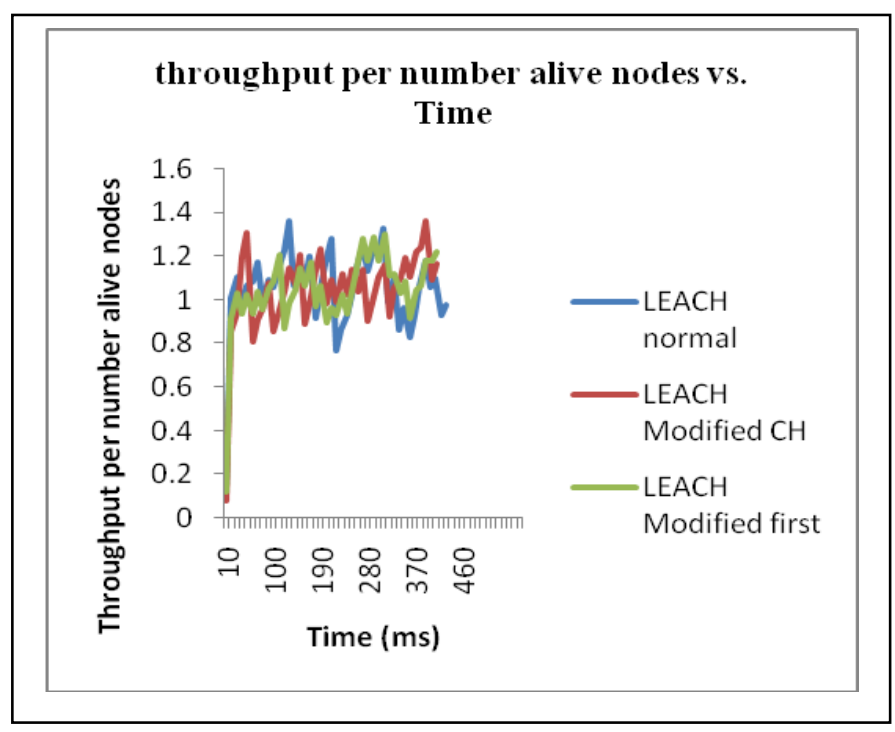

Fig. 8. . Throughput per number alive nodes

\section{CONCLUSION}

In this work, we presented the LEACH hierarchical protocol in wireless sensor network with the use of free time slots of the dead nodes. The same goal of achieving a long lifetime of wireless sensor network and a flow rate of reception elevated relative to live node. 
We also saw, through our extensive simulations we tested the stability of the life of the network, however simulation results clearly show that the line corresponding to normal LEACH protocol has a less throughput than the line of LEACH protocol modified. Therefore our technique is able to extend the performance in terms of average throughput.

\section{REFERENCES}

[1] R.SARAVANAKUMAR, S.G.SUSILA, J.RAJA "Energy Efficient Constant Cluster Node Scheduling Protocol for Wireless Sensor Networks", WSEAS TRANSACTIONS COMMUNICATIONS,pp119-128 Volume 10, April 2011.

[2] Mr. Santosh.Irappa.Shirol, Ashok Kumar, Mr. Kalmesh.M.Waderhatti, "Advanced-LEACH Protocol of Wireless Sensor Network" IJETT, Volume4 Issue6- June 2013.

[3] D. S. Kim and Y. Chung, "Self-organization routing protocol supporting mobile nodes for wireless sensor network," in Proc. of 1st Int'1 MultiSymposium on Computer and Computational Sciences (IMSCCS'06), 2006.

[4] S. A. B. Awwad, C. K. Ng, N. K. Noordin, and M. F. A. Rasid, "Cluster based routing protocol for mobile nodes in wireless sensor network," in Proc. of Int'l Symposium on Collaborative Technologies and Systems 2009, CTS'09, pp. 18-22, May 2009.

[5] G. Santhosh Kumar, V. Paul M V , and K. Poulose Jacob, "Mobility Metric based LEACH-Mobile Protocol" 16th International Conference on Advanced Computing and Communications, ADCOM pp. 248 - 253, 2008 .
[6] Ravneet Kaur1, Deepika Sharma2 and Navdeep Kaur3, "Comparative Analysis Of Leach And Its Descendant Protocols In Wireless Sensor Network", IJP2PNTT- Volume3Issue1- 2013.

[7] Karim, L. ; Nasser, N. "Energy Efficient and Fault Tolerant Routing Protocol for Mobile Sensor Network", Communications (ICC), 2011 IEEE International Conference on, On page(s):1-5

[8] Hanady M. Abdulsalam, Layla K. Kamel: W-LEACH: Weighted Low Energy Adaptive Clustering Hierarchy Aggregation Algorithm for Data Streams in Wireless Sensor Networks. ICDM Workshops 2010: 1-8

[9] Gnanambigai,Dr.N.Rengarajan,K.Anbukkarasi "Leach and Its Descendant Protocols: A Survey", International Journal of ommunication and Computer Technologies Volume 01 - No.3, Issue: 02 September 2012.

[10] Hong, J.; Kook, J.; Lee, S.; Kwon, D.; Yi, S. T-LEACH: The method of threshold-based cluster head replacement for wireless sensor networks. Inf. Syst. Front. 2009, 11, 513-521.

[11] G Haosong, L Gang, Y Yonghwan "A partition based centralized LEACH algorithm for wireless sensor netoworks using solar energy", ICHIT, Korea, Aug, 2009.

[12] Mayura Bhandarkar, "Analysis of sLEACH for improvement of network lifetime in Wireless Sensor Networks", ProQuest, 2008.

[13] Kevin Fall , Kannan Varadhan "The ns Manual" MIT, November 4, 2011.

[14] W. Heinzelman "The MIT uAMPS ns Code Extensions, Version 1.0," MIT, August 2000. 